\title{
AN INDOOR POSITIONING TECHNIQUE BASED ON A FEED-FORWARD ARTIFICIAL NEURAL NETWORK USING LEVENBERG-MARQUARDT LEARNING METHOD
}

\author{
P. Pahlavani ${ }^{\text {a,* }}$, A. Gholami ${ }^{a}$, S. Azimi ${ }^{\text {a }}$ \\ a School of Surveying and Geospatial Eng, College of Eng., University of Tehran, Tehran, Iran - \\ (pahlavani, amin.gholami, sanazazimi@ut.ac.ir)
}

KEY WORDS: Indoor positioning, Artificial Neural network, Fingerprinting technique, Received signal strength, Wireless Local Area Network

\begin{abstract}
:
This paper presents an indoor positioning technique based on a multi-layer feed-forward (MLFF) artificial neural networks (ANN). Most of the indoor received signal strength (RSS)-based WLAN positioning systems use the fingerprinting technique that can be divided into two phases: the offline (calibration) phase and the online (estimation) phase. In this paper, RSSs were collected for all references points in four directions and two periods of time (Morning and Evening). Hence, RSS readings were sampled at a regular time interval and specific orientation at each reference point. The proposed ANN based model used Levenberg-Marquardt algorithm for learning and fitting the network to the training data. This RSS readings in all references points and the known position of these references points was prepared for training phase of the proposed MLFF neural network. Eventually, the average positioning error for this network using 30\% check and validation data was computed approximately 2.20 meter.
\end{abstract}

\section{INTRODUCTION}

Recently, the positioning technique has been used widely for the location-based service (LBS) system. The global positioning system (GPS) is most popular satellite-based positioning system (Enge, 1994). However, that is not works for the indoor positioning system because of the GPS line of sight transmission method and deterioration of satellite signals at indoor environment. Unlike outdoor areas, the indoor environment has different challenges on location discovery due to the dense multipath effect and building material dependent propagation effect. Therefore other wireless positioning technologies suitable for indoor environments are necessary. Existing indoor position technologies are A-GPS, Ultrasonic Positioning Technology, Optical Location Tracking Technology, RFID, and so on (OUYANG, MA and FANG, 2008). Also, the wireless communication techniques include the wireless local area network (WLAN) (Kotanen et al., 2003), wireless sensor network (WSN) (Wong et al., 2005), radio frequency identification (RFID) (Chon et al., 2004), Bluetooth (Salazar, 2004), and so on.

There has been an increasing deployment of WLANs by many individuals and organizations inside homes, offices, buildings, and campuses. There are many positioning systems that use WLAN for estimating location such as time of arrival (TOA) (Jing et al., 2008) and time difference of arrival (TDOA)(Kolodziej and Hjelm, 2006). TOA and TDOA can use effectively for outdoor positioning system. Non line of site problem and multipath effect problem induced by building geometry, human body absorption, dynamic environments make these techniques ineffective or complex for indoor positioning systems (Sayed, Tarighat and Khajehnouri, 2005) and (Ahmad et al., 2006).

Location fingerprinting is used by most WLAN positioning systems, as it can compute accurate location estimates. The location fingerprinting can be divided into two phases: offline (calibration) phase and online (estimation) phase. In the offline phase, the received signal strength (RSS) at multiple access points (APs) are recorded to establish radio map and in the online phase, the user coordinates are estimated.

Three popular machine techniques which can be used in location fingerprinting are probabilistic method, knearest-neighbor, and neural networks.

In this article, a MLFF ANN was proposed for estimating user's coordinates in offline and online phase of location fingerprinting. The objective of this study is to estimate the location of mobile station with the small error.

ANN have capabilities for nonlinear mapping, parallel distributed processing, adaptive self-learning, data fusion, and multivariable structure (Derr and Manic, 2008), and it can reduce the time cost for location system layout, save the storage cost for radio map establishment, and enhance the real-time positioning capacity in on-line phase(Borenovic et al., 2008) and (Fang and Lin, 2008) and (Xu, Zhou and Ma, 2009).

\section{REVIEWING THE METHODS}

For indoor positioning a lot of research have been done. Tsung-Nan Lin et al compared three indoor positioning techniques including location k-nearest neighbor, artificial neural networks, and fingerprinting. In this regard, different evaluation criteria have been compared including precision, accuracy, robustness, complexity, and scalability. Their results showed that the k-nearest neighbor reaches better overall performance for the indoor positioning purpose than the other methods (Lin and Lin, 2005). Syahrulanuar NGAH et al are introduced an ANN based model for improving the positioning accuracy in local environment. The proposed ANN based model was a mapping from a set of input variables 
containing measured distances between tag and locators onto a set of two outputs tag $(x, y)$. Proposed ANN based model supported by the good training data to estimate the locations of tag with small error and reduce the computation time as well. The results of evaluation criteria including accuracy, precision, and computational time were shown that the ANN based model provided better positioning accuracy and precision, compared to the Genetic Algorithm (GA) and the Particle Swarm Optimization (PSO). Also, the computation time of ANN based model is reduced $10 \%$ and $20 \%$ in comparison to those by GA and PSO, respectively (Ngah et al., 2009). An active RFID and artificial neural networks were used by Santos Martínez Sala et al. for developing a real-time LBS. Their model-was based on a Multilayer Perceptron trained and evaluated by a radio map for learning to compute the tags position. By simulation, the proper MLP architecture and the mean error positioning estimation depended on the number of Readers. By 8 Readers deployed in an indoor area of $576 \mathrm{~m}^{2}$, an error less than $1.75 \mathrm{~m}$ in the $75 \%$ of the target area was achieved (Sala, Quiros and Lopez, 2010). The ZigBee wireless sensor network using ANN was proposed by Rey-Chue Hwang et al. for locating the high accuracy position when the signal strengths are unstable. They discover that the accuracy of polar form is better than the rectangular one. Additionally, ANN approximates the parameters of radio signal power delay profile and accordingly calculates the location of the user (Hwang et al., 2011). A hybrid Genetic Algorithm-Back Propagation Neural Network, GA-BP $\mathrm{NN}$, was proposed by algorithMiao et al. They proposed a 2D RFID-based indoor positioning technique. Their show that in an indoor environment, the ANN can lessen problems occurred by the complexity and variation in the radio signal propagation (Kehua, YaoDong and Xiao, 2011).

\subsection{Fingerprinting}

Most of the indoor RSS-based WLAN positioning systems utilize the fingerprinting technique for acquiring the RSS and position relation for computing a more accurate estimation of user's position. The location fingerprinting can be divided into two phases: offline (calibration) phase and online (estimation) phase

In the offline phase, the fingerprinting technique generally requires measurement of RSS or the other nongeometric features from different APs by WLAN-enabled mobile device at the desired known positions (reference points) to form a database of location fingerprints. The database of location fingerprinting is known as the radio map on the server. In the other hand, Offline phase is the training period that collects RSS data at the area of interest and preprocesses them for estimating the user's position in the online phase. The required time for the training depends on the size of the survey site and the resolution of surveying. The time interval of RSS measuring and direction of antenna inside the device affects the RSS readings. Hence, RSS readings are sampled at a regular time interval and specific orientation at each reference point. During the online phase, the device collects online RSS readings from device location in known orientation that are then used together with the fingerprint database to estimate the device's location.

The accuracy of fingerprinting techniques is highly dependent on the density of the signal database. Building and maintaining a high-density database are not easy, because of two reasons: Firstly, building a high-density fingerprint database is the labor intensive, expensive, and even impossible in some cases. Secondly, maintaining a large signal database is expensive (Guzmán-Quirós et al., 2015).

\subsection{Artificial Neural Network model}

The objective of this work is to estimate the locations of the users with small error. To achieve this objective, ANN based model has been choose. ANN based model has the learning ability and can achieve high accuracy and precision for indoor positioning with less computational time.

ANN is composed of elements operating in parallel and has a specific knowledge for specific problems. In this regard, the computational time can be extremely reduced. Also, ANN can overcome the problems where normal computation cannot solve (Ngah et al., 2009).

Multi layer feed forward (MLFF) neural networks are contained of three layer types: the input layer, the output layer, and the hidden layers between them. One of the most popular algorithm for training the MLFF neural networks is back propagation(Yu and Wilamowski, 2011). Figure 1 shows a typical feed-forward neural network.

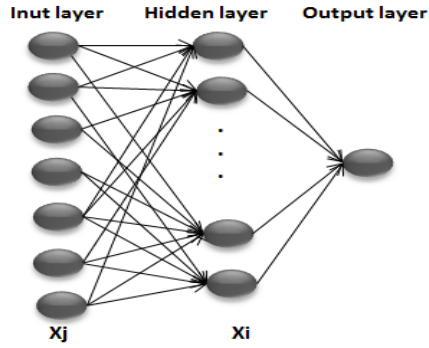

Figure 1. Typical feed-forward neural network composed of three layers

An activation function is assigned to the neurons of input, hidden, and output layers. An activation function compute the output value for an specific neuron based on the incomming values of the previous neurons. The previous neurons connecting to the mentioned neuron by the weighted channels.

\subsection{Levenberg-Marquardt Algorithm}

The back-propagation algorithm is the popular algorithm for training the neural networks. But, because of slow convergence of it, it becomes inefficient algorithm for training the neural networks. In this regard, the Levenberg-Marquardt algorithm was proposed as a prominent alternative to the Gauss-Newton method for finding the minimum of a function.

The Levenberg-Marquardt algorithm can be considered as a combination of the Gauss-Newton algorithm and the error back-propagation method. The LevenbergMarquardt algorithm has the high speed advantage of the Gauss-Newton algorithm and the stability of the steepest descent method (Hagan and Menhaj, 1994), while gaining more robustness because of the good convergences ability 
in complex error surfaces. However the LevenbergMarquardt algorithm is faster than the back-propagation algorithm.

The main idea of the Levenberg-Marquardt algorithm is to perform a flexible training process. In the other hand, this algorithm applies the steepest descent algorithm in the areas representing sophisticated curvature. This algorithm makes a quadratic approximation to expedite the training procedure, when the local curvature satisfies the GaussNewton algorithm (Yu and Wilamowski, 2011).

The Levenberg-Marquardt algorithm solves the following equation to approximate a function ( $\mathrm{Yu}$ and Wilamowski, 2011):

$$
\left(\mathrm{J}^{\mathrm{T}} \mathrm{J}+\lambda \mathrm{I}\right) \delta=\mathrm{J}^{\mathrm{T}} \mathrm{E}
$$

where $\quad \mathrm{J}=$ the Jacobian

$\lambda=$ the Levenberg's adjustment factor

$\delta=$ the weight update vector

$\mathrm{E}=$ the final error vector of the network

$\mathbf{J}^{\mathrm{T}} \mathbf{J}=$ approximated Hessian matrix

$\delta$ obtains and determines the required changes in the network weights to achieve a better solution. $\mathrm{E}$ is the final error vector of the network containing the output errors for all input vectors used to train the network. The $\lambda$ adjustment factor is adjusted at each iteration, and it used to control the optimization process of the network In the neural network case, the Jacobian matrix is an Mby-W matrix, where $\mathrm{M}$ is the size of each patterm in the training set and $\mathrm{W}$ is the total number of parameters to be obtained including weights and biases of the network, and so the jacobian would be as follows ( $\mathrm{Yu}$ and Wilamowski, 2011):

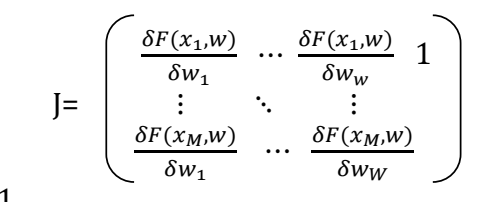

$F\left(x_{i}, w\right)=$ the activation function for the $i$-th input pattern of the training set $\mathrm{w} j=$ the $j$-th element of the weight vector $w$ between nodes of the current layer in the network

For obtaining the training parameters, the LevenbergMarquardt algorithm initializes the adjustment factor $\lambda$ with a small value such as 0.1 and then this value is added to the approximate Hessian diagonal members. Then, a decomposition is performed to solve the LevenbergMarquardt equation, unless the aproximated Hessian become singular so that the inverse can not be computed. Even in this case, there are other types of decomposition that allows the equation to be solved. By solving the equation, the weights $w$ are apdated according to the calculated update vector $\delta$. After updating the weights of the network, the residual values for all input patterns of the network is recalculated and if the sum of squared of this residuals has decreased, current iteration stops with reducing the $\lambda$ value. In the opposite case, the algorithm discards the computed weights and repeates with a higher value for $\lambda$. The mentioned changes in the $\lambda$ is applied by adjustment factor $\mathrm{v}$, commonly initialized by the value of 10. If algorithm determines to increase $\lambda$, it multiplies $\lambda$ by v. Otherwise, it divides $\lambda$ by v. This process continues to onserve reduction of output errors to cease the iteration. This process is shown in Figure 2.

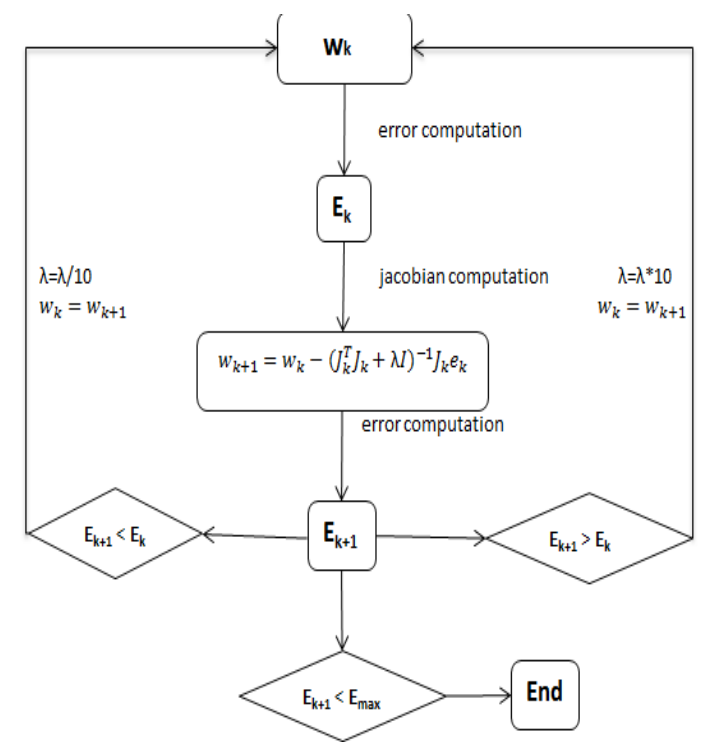

Figure 2. Updating the weight vector through implementaion of Levenberg-Marquardt algorithm

\section{PROPOSED METHOD}

As mentioned before, the collected RSS readings were done in the offline phase of location finger printing. The dynamic multi-path effect, interference with adjacent receivers, different types of wireless network cards, connection state of receivers, and many other unpredictable factors influence the WLAN signal distributions in indoor environment (Alvarez-Cabanillas, 2006). In this study, for decreasing these effects, the RSSs were collected in several directions and several time intervals.

As shown in Figure 3, RSS readings in all refrences points and the khown position of these refrences points were prepared for training phase of the proposed the MLFF ANN.

By inputing this information to the network, weights and biases of the networked would be achieved by the Levenberg-Marquardt back-propagation algorithm and the structure of the network was formed. Hence, the system would be considered ready to operate in the online phase of location finger printing. In the online phase, the RSS readings of user were entered to MLFF ANN based model. This model was trained in offline phase and the location of user was computed.

As mentioned earlier, the objective of this study is to determine the location of mobile station with the small error. Briefly, the process flowchart of this proposed model is shown in Figure 4. 


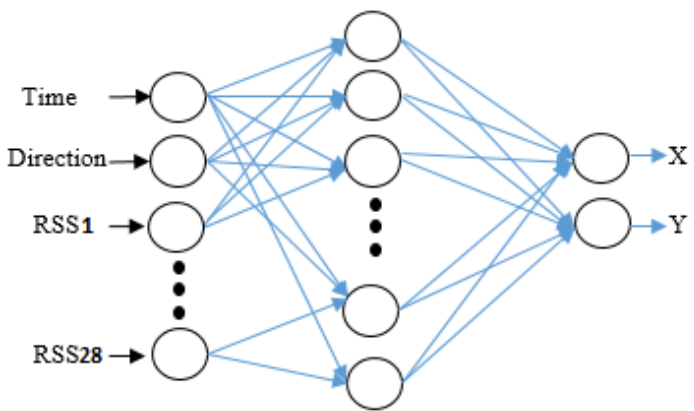

Figure 3. Multilayer perceptron:proposed model
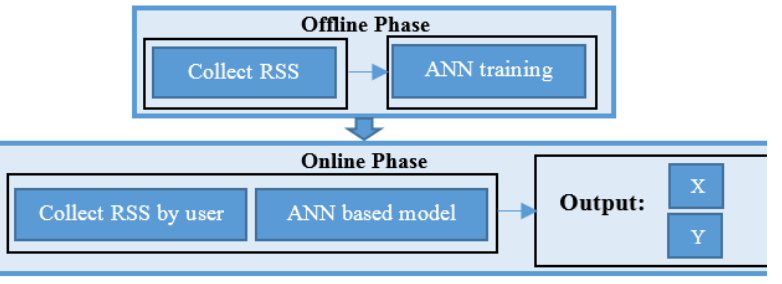

Figure 4. Process flowchart of the proposed method

\section{IMPLEMENTATION}

\subsection{Data set}

The plan of study area is depicted in Figure 5. In this study area, 28 APs and 67 reference points was considered. For surveying this study area, the local coordination system was considered. Also, the north direction was assumed local in this local coordination system. The RSS readings are collected at four common directions namely North, East, South, and West and in two periods of time, i.e. Morning and Evening, for each references point. Generally, 536 points was collected in the mentioned study area.
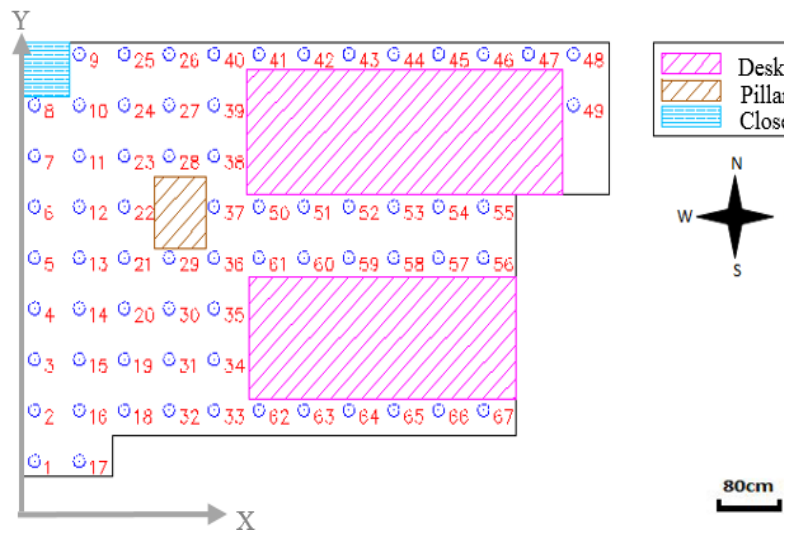

Figure 5. The plan of selected study area

\subsection{Results}

In this study, ASUS laptop was used for collecting the RSS readings. The profile of this laptop is described in Table 1. The proper C\# code was implemented in this laptop for collecting RSS readings in references points and inserting them with their directions and their time intervals to MySQL database. Then, this information saved in this database. In Figure 6, by pressing 'insert to DB' click in mentioned C\# code, the RSS in one reference point at one direction in one time interval was collected and inserted to MySQL database.

\begin{tabular}{|c|c|c|c|c|}
\hline \multicolumn{2}{|l|}{ MAC Address } & SSID & Channel & Authertication \\
\hline \multicolumn{2}{|c|}{$00.23 .69 .78 .18 \mathrm{fd}$} & Inkays & 6 & $58 \%$ \\
\hline \multicolumn{2}{|c|}{ 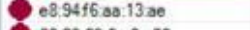 } & GEO Center & 7 & $100 \%$ \\
\hline \multicolumn{2}{|c|}{$00.23 .69 .6 \mathrm{c} \cdot 6 \mathrm{a} \cdot 08$} & geote acir & 4 & $70 \%$ \\
\hline \multirow{2}{*}{\multicolumn{2}{|c|}{$\begin{array}{l}14 f 2.6 d 10.0 a \cdot 54 \\
50.6740 .28: 11: 70\end{array}$}} & Lab_008 & 2 & $48 \%$ \\
\hline & & ZyXEL & 6 & $0 \%$ \\
\hline \multicolumn{2}{|c|}{$\begin{array}{l}50.6740 .28 \cdot 1 f: 70 \\
c 8: 3 a: 35 \cdot 4 a \cdot c 0=88\end{array}$} & Geodesy-A & 6 & $72 \pi$ \\
\hline \multicolumn{2}{|c|}{$\begin{array}{l}c 8: 3 a: 35 \cdot 4 a \cdot c 0088 \\
c 8: 4 c: 75 \cdot 20: 0 e \cdot c 3\end{array}$} & indut acir & 11 & $0 \%$ \\
\hline & dink & 1 & $48 \%$ \\
\hline \multicolumn{2}{|c|}{ 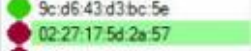 } & $\begin{array}{l}\text { HPFOF955 } \\
\text { PElab }\end{array}$ & 10 & $54 \%$ \\
\hline \multicolumn{2}{|c|}{ 00:1f. $17: 8 d .4 b$ bc } & & 11 & $52 \%$ \\
\hline \multirow{2}{*}{\multicolumn{2}{|c|}{$18.07: 28.54 .58 .3 \mathrm{~d}$}} & Indutacr & 1 & $42 \%$ \\
\hline & & $a b$ & 6 & $42 \%$ \\
\hline \multicolumn{5}{|l|}{$<$} \\
\hline \multicolumn{5}{|l|}{ Inout } \\
\hline \multirow{3}{*}{$\begin{array}{l}\checkmark \text { Wosth } \\
\square \text { Esst } \\
0 \text { South } \\
\square \text { West }\end{array}$} & Moming & \multirow{2}{*}{ Oftine } & & \\
\hline & $\checkmark$ Eveing & & & \\
\hline & & Insert to $D B$ & & \\
\hline \multicolumn{2}{|l|}{ (A) } & & & \\
\hline
\end{tabular}

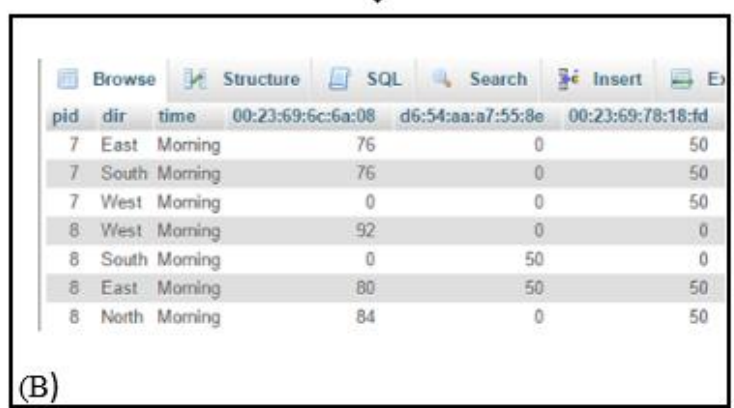

Figure 6. A) Proposed C\# code for collecting, B) Insertion of RSS to MySQL database

The input data of training phase of the proposed MLFF ANN were normalized. Then, the training phase of this network was implemented in MATLAB. Evaluation of percentage of training data to MSE was shown in Figure 7. In this graph, the best MSE (or minimum MSE) belongs to $70 \%$ training data and $30 \%$ check and validation data. Thus, $70 \%$ of total data were considered as the training data and $30 \%$ of the total data were considered as the check and validation data.

In Figure 8, the graph of number of nerouns in hidden layer of proposed MLFF ANN to MSE for check points was drawn. According to this graph, the minimum value of MSE belongs to 7 neurons. Because of the best MSE result for 7 neurons, this number of neurons were considered for hidden layer in training phase of the proposed network.

\begin{tabular}{|l|c|}
\hline Model & Asus, x550c \\
\hline CPU & $\begin{array}{c}\text { Intel Core i5 3337U } \\
1.80 \mathrm{GHz} \text { up to 2.70 GHz }\end{array}$ \\
\hline Memory & 6 GB RAM \\
\hline Platform & Windows 8 \\
\hline $\begin{array}{l}\text { Wireless networking } \\
\text { support }\end{array}$ & $802.11 \mathrm{n}$ \\
\hline
\end{tabular}

Table 1. The profile of Asus laptop was used for collecting the RSS readings 


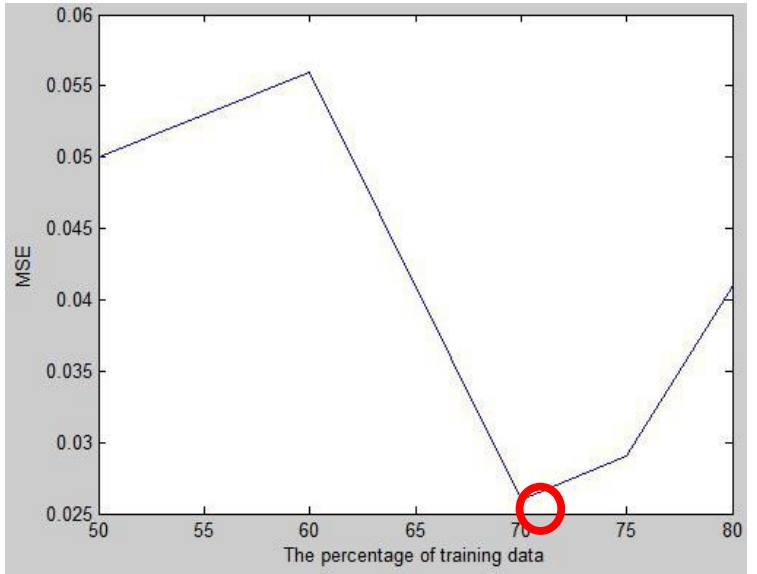

Figure 7. Evaluating the percentage of training data to MSE

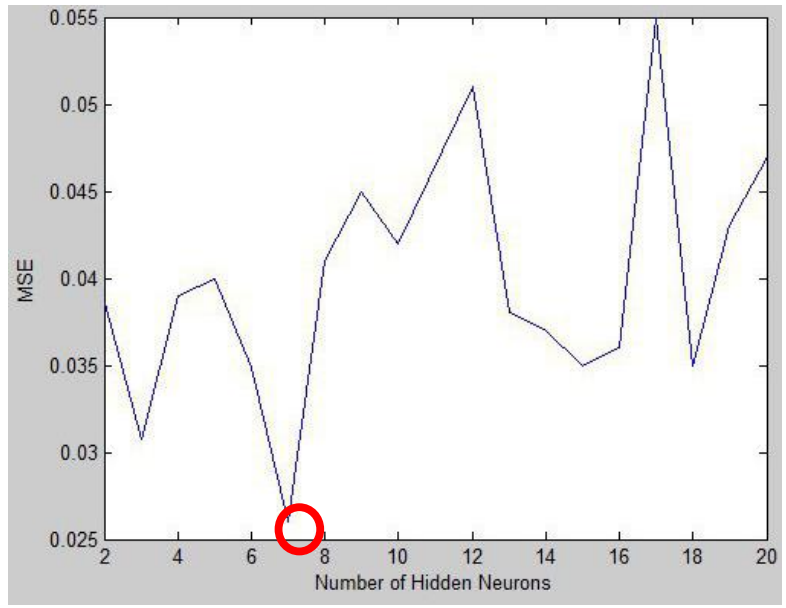

Figure 8. Evaluating the number of nerouns to MSE

In Figure 9, the regression diagrams achieved by the designed neural network are shown and in Figure 10 the performance (MSE) of the proposed neural network for this Levenberg-Marquardt training phase is shown.

After completaion of the training phase, the system became ready to operate in the online phase of location fingerprinting.

In the online phase, RSSs were collected in several unknown points. These collected RSSs with their directions and their time intervals were considered as the entered information in the proposed MLFF ANN. The output of this network was determined as the location of these unknown points. For one point with actual coordinates $(5.6,4)$, the computed coordinate became $(5.53,3.95)$ in the proposed MLFF ANN. So, the RMSE of this point became $0.077 \mathrm{~m}$. For another point with actual coordinates $(0.8,0.8)$, the computed coordinate became $(5.23,3.75)$ in this network and the RMSE of this point became $5.32 \mathrm{~m}$. The MSE for the check data was achieved 0.026 . The training data correlation became 0.96 and the total correlation of data became 0.9. Eventually, the average positioning error for the proposed MLFF ANN with $30 \%$ check and validation data was computed 2.20 $\mathrm{m}$.
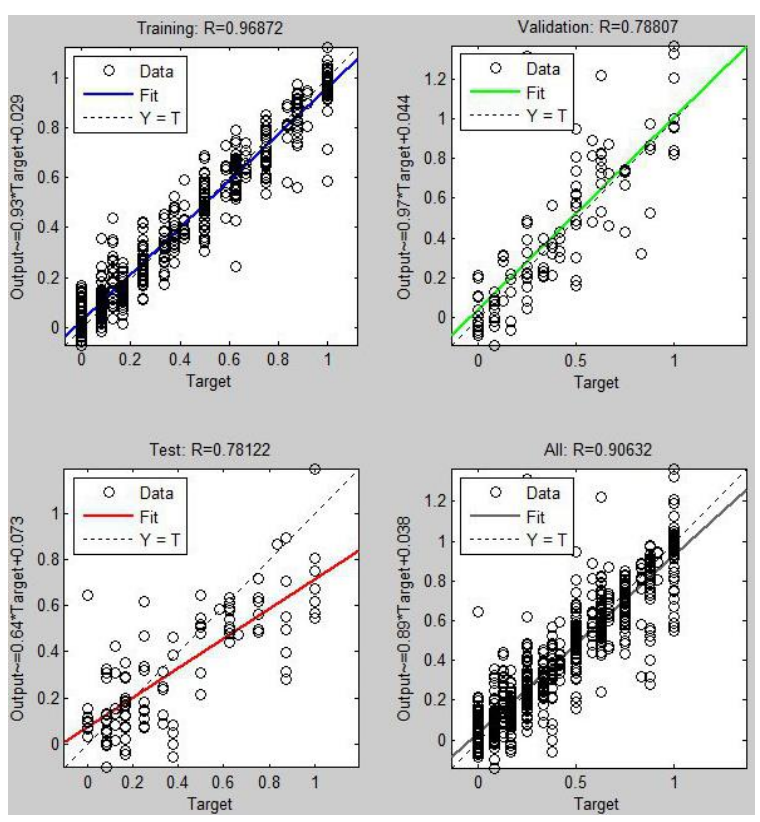

Figure 9 . The regression diagrams achieved by the designed neural network.

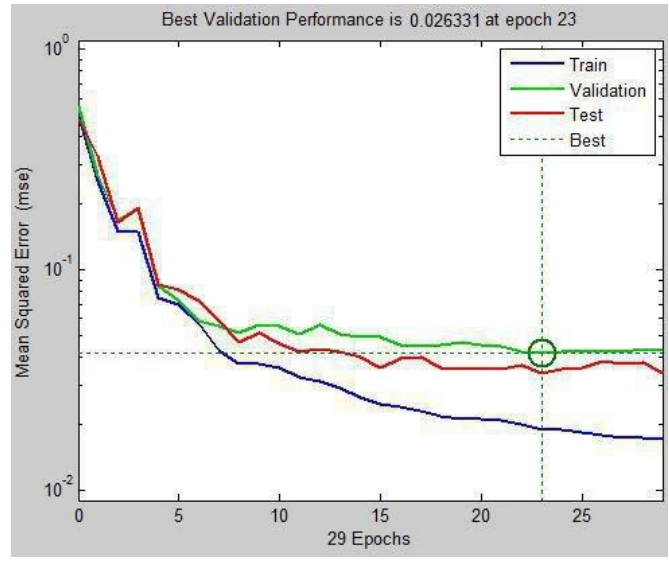

Figure 10. The performance (MSE) of the proposed neural network.

\section{CONCLUSION}

As the demand of indoor Location-Based Services (LBSs) increases, there is a growing interest in developing an accurate indoor positioning. The indoor environments are highly structured and more complicated than the outdoors environment. Global positioning systems have difficulties for positioning inside buildings; so, other techniques and methods are required for indoor positioning. This study presents the implementation of location fingerprinting methods for indoor positioning.

Location fingerprinting is used by most WLAN positioning systems for computing the accurate location estimations. Offline and online phase are two phases of the location fingerprinting. In this study, MLFF ANN was proposed for estimating user's coordinates in offline and online phase of location fingerprinting. In the offline phase, RSSs were collected at four common directions and in two time intervals and the Levenberg-Marquardt backpropagation algorithm was considered for training phase 
of the proposed MLFF ANN. Seven neurons for the hidden layer and $70 \%$ training data and $30 \%$ check and validation data were considered in training phase. In the online phase, the output of the proposed MLFF ANN was the location of user. Compared to the other researches, the results of this study shown considerable reduction error for the location of user. On the other hand, the average positioning error for the proposed network consisting of $30 \%$ check and validation data was achieved approximately $2.20 \mathrm{~m}$.

In future work, the proposed network can be implemented in big area and more periods of time can be considered. The RMSE can be reduced with these conditions. Distance difference of APs and references point for APs with known coordinates can have significant impact on decreasing of the noise.

\section{REFERENCES}

Ahmad, U., A. Gavrilov, S. Lee \& Y.-K. Lee., 2006. Modular multilayer perceptron for WLAN based localization. In The 2006 IEEE International Joint Conference on Neural Network Proceedings, pp. 3465-3471. IEEE.

Alvarez-Cabanillas, M. A., 2006. Indoor propagation of diffracted signals. In 2006 IEEE Antennas and Propagation Society International Symposium, pp. 2181-2184. IEEE.

Borenovic, M., A. Neskovic, D. Budimir \& L. Zezelj., 2008. Utilizing artificial neural networks for WLAN positioning. In 2008 IEEE 19th International Symposium on Personal, Indoor and Mobile Radio Communications, pp. 1-5. IEEE.

Chon, H. D., S. Jun, H. Jung \& S. W. An., 2004. Using RFID for accurate positioning. Positioning, 1.

Derr, K. \& M. Manic., 2008. Wireless based object tracking based on neural networks. In 2008 3rd IEEE Conference on Industrial Electronics and Applications, pp. 308-313. IEEE.

Enge, P. K., 1994. The global positioning system: Signals, measurements, and performance. International Journal of Wireless Information Networks, 1, pp. 83-105.

Fang, S.-H. \& T.-N. Lin., 2008. Indoor location system based on discriminant-adaptive neural network in IEEE 802.11 environments. IEEE Transactions on Neural networks, 19, pp.19731978.

Guzmán-Quirós, R., A. Martínez-Sala, J. L. GómezTornero \& J. García-Haro., 2015. Integration of directional antennas in an RSS fingerprintingbased indoor localization system. Sensors, 16, 4 .

Hagan, M. T. \& M. B. Menhaj., 1994. Training feedforward networks with the Marquardt algorithm. IEEE transactions on Neural Networks, 5, pp. 989-993.

Hwang, R.-C., P.-T. Hsu, J. Cheng, C.-Y. Chen, C.-Y. Chang \& H.-C. Huang., 2011. The indoor positioning technique based on neural networks. In Signal Processing, Communications and Computing (ICSPCC), 2011 IEEE International Conference on, pp. 1-4. IEEE.

Jing, L., P. Liang, C. Maoyong \& S. Nongliang., 2008. Super-resolution time of arrival estimation for indoor geolocation based on IEEE $802.11 \mathrm{a} / \mathrm{g}$. In Intelligent Control and Automation, 2008. WCICA 2008. 7th World Congress on, pp. 66126615. IEEE.

Kehua, M., C. YaoDong \& M. Xiao., 2011. An indoor positioning technology based on GA-BP neural network. In Computer Science \& Education (ICCSE), 2011 6th International Conference on, pp. 305-309. IEEE.

Kolodziej, K. W. \& J. Hjelm., 2006. Local positioning systems: LBS applications and services. CRC press.

Kotanen, A., M. Hannikainen, H. Leppakoski \& T. Hamalainen., 2003. Positioning with IEEE $802.11 \mathrm{~b}$ wireless LAN. In Personal, Indoor and Mobile Radio Communications, 2003. PIMRC 2003. 14th IEEE Proceedings on, pp. 22182222. IEEE.

Lin, T.-N. \& P.-C. Lin., 2005. Performance comparison of indoor positioning techniques based on location fingerprinting in wireless networks. In 2005 international conference on wireless networks, communications and mobile computing, pp. 1569-1574. IEEE.

Ngah, S., H. Zhu, K.-t. Chen, Y. Tanabe \& T. Baba., 2009. Artificial neural network based model for local position systems.

OUYANG, Y.-m., Z.-q. MA \& R.-s. FANG., 2008. Design and realization of genetic-neural network Based on MATLAB. Information Technology, 6, 023.

Sala, A. S. M., R. G. Quiros \& E. E. Lopez., 2010. Using neural networks and Active RFID for indoor location services. In Smart Objects: Systems, Technologies and Applications (RFID Sys Tech), 2010 European Workshop on, pp. 1-9. VDE.

Salazar, A. S., 2004. Positioning Bluetooth ${ }^{\circledR}$ and Wi-FiTM systems. IEEE Transactions on Consumer Electronics, 50, pp. 151-157.

Sayed, A. H., A. Tarighat \& N. Khajehnouri., 2005. Network-based wireless location: challenges faced in developing techniques for accurate wireless location information. IEEE signal processing magazine, 22, pp.24-40.

Wong, K.-F., I. W. Tsang, V. Cheung, S.-H. Chan \& J. T. Kwok., 2005. Position estimation for wireless sensor networks. In GLOBECOM'05. IEEE Global Telecommunications Conference, 2005., 5 pp.-2776. IEEE.

Xu, Y., M. Zhou \& L. Ma., 2009. WiFi indoor location determination via ANFIS with PCA methods. In 2009 IEEE International Conference on Network Infrastructure and Digital Content, pp. 647-651. IEEE.

Yu, H. \& B. M. Wilamowski., 2011. Levenbergmarquardt training. Industrial Electronics Handbook, 5, 1 . 